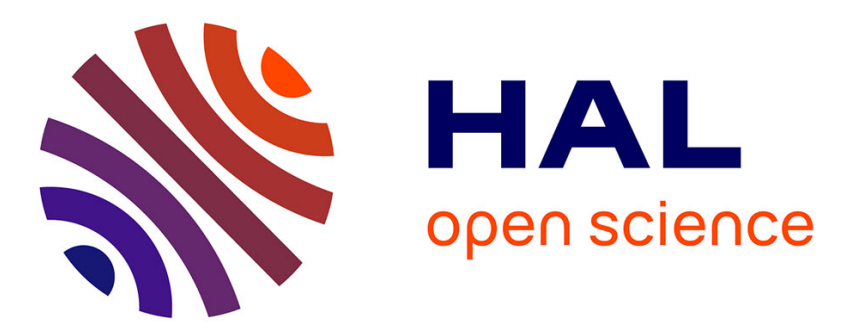

\title{
LES of the flow past a circular cylinder using a multiscale discontinuous Galerkin method
}

\author{
Marta de La Llave Plata, Fabio Naddei, Vincent Couaillier
}

\section{To cite this version:}

Marta de La Llave Plata, Fabio Naddei, Vincent Couaillier. LES of the flow past a circular cylinder using a multiscale discontinuous Galerkin method. 5th Internationnal conference on Turbulence and Interactions, TI 2018, Jun 2018, (MARTINIQUE), France. hal-02491887

\section{HAL Id: hal-02491887 \\ https://hal.science/hal-02491887}

Submitted on 26 Feb 2020

HAL is a multi-disciplinary open access archive for the deposit and dissemination of scientific research documents, whether they are published or not. The documents may come from teaching and research institutions in France or abroad, or from public or private research centers.
L'archive ouverte pluridisciplinaire HAL, est destinée au dépôt et à la diffusion de documents scientifiques de niveau recherche, publiés ou non, émanant des établissements d'enseignement et de recherche français ou étrangers, des laboratoires publics ou privés. 


\title{
LES of the flow past a circular cylinder using a multiscale discontinuous Galerkin method
}

\author{
Marta de la Llave Plata ${ }^{1}$, Fabio Naddei ${ }^{1}$, and Vincent Couaillier ${ }^{1}$ \\ ${ }^{1}$ ONERA - Université Paris-Saclay \\ 92320 Châtillon, France \\ marta.de_la_llave_plata@onera.fr, \\ WWW home page: https://www.onera.fr/
}

\begin{abstract}
The variational multiscale (VMS) approach based on a modal discontinuous Galerkin (DG) method is used to perform LES of the sub-critical flow past a circular cylinder at Reynolds numbers 20000 and 140000 , based on the cylinder diameter. The potential of using padaption in combination with DG-VMS is illustrated for the case at $R e=140000$ by considering a non-uniform distribution of the polynomial degree based on a recently developed error estimation strategy [15].
\end{abstract}

Keywords: discontinuous Galerkin, p-adaptivity, large-eddy simulation, variational multiscale approach.

\section{Introduction}

The study of flows past cylinders is of relevance to many engineering applications, such as noise prediction in aircraft landing gears. A characteristic feature of bluff body aerodynamics is the appearance of a large-scale vortex shedding in the near wake which can lead to flow-induced sound and vibration of the structure. The ability to accurately predict this phenomenon across a wide range of Reynolds numbers using numerical methods is especially challenging, in particular because the location of the separation point on the cylinder wall is very sensitive to small disturbances in the flow. On the other hand, the geometrical representation of the cylinder adds an extra level of complexity in the context of high-order methods, due to the necessity to use large curved elements around the wall when the order of the polynomial approximation is increased (p-refinement).

In this work, the scale-resolving capabilities of the DG solver Aghora [4, 18] are illustrated by performing LES of the flow past a cylinder in the subcritical regime at $R e=20000$ and 140000 . The subgrid-scale (SGS) modelling approach adopted here is based on the VMS approach developed in [5] in the context of a modal DG method (DG-VMS). The potential of using p-adaptation for LES is demonstrated for the case at $R e=140000$ by defining a non-uniform distribution of local polynomial degrees based on a recently developed error estimation strategy [15].

A limited number of LES simulations of this configuration have been reported in the literature for these two Reynolds numbers. We can cite the work presented 
in $[19,14,13]$ for $R e=20000$ and in $[2,9]$ for $R e=140000$ based on second-order finite-volume methods (FVM) and different SGS models, and grid resolutions. The outcome of our DG-VMS simulations is compared with the high-resolution LES results reported by Lysenko et al. [13] for $R e=20000$, and by Breuer [2] for $R e=140000$ using low-dissipation second-order FVM. The material presented in these two articles is well documented and, in our view, constitutes a valuable reference to assess the performance of the present approach.

\section{Governing equations}

Let $\Omega \subset \mathbb{R}^{3}$ be a bounded domain. The compressible Navier-Stokes (N-S) equations, with appropriate boundary conditions in $\partial \Omega$, read

$$
\frac{\partial \mathbf{u}}{\partial t}+\nabla \cdot\left(\mathcal{F}_{c}(\mathbf{u})-\mathcal{F}_{v}(\mathbf{u}, \nabla \mathbf{u})\right)=0, \text { in } \Omega
$$

where $\mathbf{u}=(\rho, \rho \mathbf{v}, \rho E)^{T}$ is the vector of conservative variables. The vectors $\mathcal{F}_{c}$, and $\mathcal{F}_{v}$ are the convective and viscous fluxes, respectively,

$$
\mathcal{F}_{c}=(\rho, \rho \mathbf{v} \otimes \mathbf{v}+p \overline{\overline{\mathbf{I}}},(\rho E+p) \mathbf{v})^{T}, \mathcal{F}_{v}=(0, \overline{\overline{\boldsymbol{\tau}}}, \overline{\overline{\boldsymbol{\tau}}} \cdot \mathbf{v}-\mathbf{q})^{T}
$$

In (2), $\overline{\overline{\boldsymbol{\tau}}}$ represents the shear-stress tensor, given by

$$
\overline{\overline{\boldsymbol{\tau}}}=\mu\left(2 \overline{\overline{\mathbf{S}}}-\frac{2}{3}(\nabla \cdot \mathbf{v}) \overline{\overline{\mathbf{I}}}\right) \quad \text { with } \quad \overline{\overline{\mathbf{S}}}=\frac{1}{2}\left(\nabla \mathbf{v}+\nabla \mathbf{v}^{T}\right)
$$

$\mu$ is the dynamic viscosity and $\overline{\overline{\mathbf{S}}}$ the rate-of-strain tensor. The heat-flux vector is written as $\mathbf{q}=-k \nabla T$, with $T$ the temperature and $k$ the thermal diffusivity.

\section{The DG-VMS formulation}

Let $\Omega_{h}$ be a shape-regular partition of the domain $\Omega$, into $N$ non-overlapping and non-empty cells $\kappa$ of characteristic size $h$. We also define the sets $\mathcal{E}_{i}$ and $\mathcal{E}_{b}$ of interior and boundary faces in $\Omega_{h}$, respectively, such that $\mathcal{E}_{h}=\mathcal{E}_{i} \cup \mathcal{E}_{b}$. Let $\mathcal{V}_{h}^{p}=\left\{\phi \in L^{2}\left(\Omega_{h}\right):\left.\phi\right|_{\kappa} \in \mathcal{P}^{p}(\kappa), \forall \kappa \in \Omega_{h}\right\}$ be the functional space of piecewise polynomials of degree at most $p$, and $\left(\phi_{\kappa}^{1}, \ldots, \phi_{\kappa}^{N_{p}}\right) \in \mathcal{P}^{p}(\kappa)$ a hierarchical and orthonormal modal basis of $\mathcal{V}_{h}^{p}$, of dimension $N_{p}$, confined to $\kappa$ [1]. The solution in each element is thus expressed as a linear expansion of basis functions, the coefficients of which constitute the degrees of freedom (DOFs) of the problem at hand.

The LES technique used in this research is based on the projection of the N-S equations onto a the functional space $\mathcal{V}_{h}^{p}$. This projection operation implicitly defines a partitioning of the solution such that a turbulent field $\mathbf{u}$ is decomposed 
into its resolved, $\mathbf{u}_{h}$, and unresolved components, $\mathbf{u}-\mathbf{u}_{h}$. The variational form of the LES equations thus reads: find $\mathbf{u}_{h}$ in $\mathcal{V}_{h}^{p}$ such that $\forall \phi_{h} \in \mathcal{V}_{h}^{p}$ we have

$$
\frac{\partial}{\partial t} \int_{\Omega_{h}} \phi_{h} \mathbf{u}_{h} d V+\mathcal{L}_{c}\left(\mathbf{u}_{h}, \phi_{h}\right)+\mathcal{L}_{v}\left(\mathbf{u}_{h}, \phi_{h}\right)=\mathcal{L}_{s g s}\left(\mathbf{u}, \mathbf{u}_{h}, \phi_{h}\right),
$$

where we have used the definition of the $L^{2}$-projection, i.e. $\int_{\Omega_{h}}\left(\mathbf{u}-\mathbf{u}_{h}\right) \phi_{h}=$ $0, \forall \phi_{h} \in \mathcal{V}_{h}^{p}$. In Eqn. (4) $\mathcal{L}_{c}$ and $\mathcal{L}_{v}$ represent the weak form of the convective and viscous terms, respectively. The term $\mathcal{L}_{\text {sgs }}$ on the right-hand-side of Eqn. (4), is the variational form of the SGS residual representing the effect of the unresolved scales $\mathbf{u}-\mathbf{u}_{h}$ on the resolved field $\mathbf{u}_{h}$.

We now introduce the following notation: for a given interface in $\mathcal{E}_{i}$ we define the average operator $\{\mathbf{u}\}=\left(\mathbf{u}^{+}+\mathbf{u}^{-}\right) / 2$ and the jump operator $\llbracket \mathbf{u} \rrbracket=\mathbf{u}^{+} \otimes \mathbf{n}-$ $\mathbf{u}^{-} \otimes \mathbf{n}$, where $\mathbf{u}^{+}$and $\mathbf{u}^{-}$are the traces of the variable vector $\mathbf{u}$ at the interface between elements $\kappa^{+}$and $\kappa^{-}$, and $\mathbf{n}$ denotes the unit outward normal vector to an element $\kappa^{+}$. The DG discretization of the convective terms reads

$$
\begin{aligned}
\mathcal{L}_{c}\left(\mathbf{u}_{h}, \phi_{h}\right) \cong & -\int_{\Omega_{h}} \mathcal{F}_{c}\left(\mathbf{u}_{h}\right) \cdot \nabla_{h} \phi_{h} d V \\
& +\int_{\mathcal{E}_{i}} \llbracket \phi_{h} \rrbracket \mathbf{h}_{c}\left(\mathbf{u}_{h}^{+}, \mathbf{u}_{h}^{-}, \mathbf{n}\right) d S+\int_{\mathcal{E}_{b}} \phi_{h}^{+} \mathcal{F}_{c}\left(\mathbf{u}_{b}\right) \cdot \mathbf{n} d S
\end{aligned}
$$

where the boundary values $\mathbf{u}_{b}=\mathbf{u}_{b}\left(\mathbf{u}_{\mathbf{h}}^{+}, \mathbf{u}_{\text {ext }}, \mathbf{n}\right)$, with $\mathbf{u}_{\text {ext }}$ a reference external state, are computed so that the boundary conditions are satisfied on $\mathcal{E}_{b}$. In this paper, a modified version of the local Lax-Friedrichs (LLF) flux and the Roe scheme have been employed for the simulations at the lower and higher Reynolds numbers, respectively,

$$
\mathbf{h}_{c}\left(\mathbf{u}_{h}^{+}, \mathbf{u}_{h}^{-}, \mathbf{n}\right)=\frac{1}{2}\left(\mathcal{F}_{c}\left(\mathbf{u}_{h}^{+}\right) \cdot \mathbf{n}+\mathcal{F}_{c}\left(\mathbf{u}_{h}^{-}\right) \cdot \mathbf{n}+\alpha \mathcal{D}\left(\mathbf{u}_{h}^{+}, \mathbf{u}_{h}^{-}, \mathbf{n}\right)\right)
$$

where $\mathcal{D}\left(\mathbf{u}_{h}^{+}, \mathbf{u}_{h}^{-}, \mathbf{n}\right)$ is the upwinding dissipation function associated with the selected numerical flux. This is scaled by a coefficient $\alpha$ to calibrate the amount of numerical dissipation introduced. Based on numerical experiments, $\alpha=0.1$ in this study. Indeed, for this value the simulation remains stable while minimizing the numerical dissipation introduced via the numerical flux.

The discretization of the viscous terms is performed using the symmetric interior penalty (SIP) method proposed by Hartmann and Houston [8],

$$
\begin{aligned}
& \mathcal{L}_{v}\left(\mathbf{u}_{h}, \phi_{h}\right) \cong \int_{\Omega_{h}} \mathcal{F}_{v}\left(\mathbf{u}_{h}, \nabla_{h} \mathbf{u}_{h}\right) \cdot \nabla_{h} \phi_{h} d V \\
& -\int_{\mathcal{E}_{i}} \llbracket \phi_{h} \rrbracket\left\{\mathcal{F}_{v}\left(\mathbf{u}_{h}, \nabla_{h} \mathbf{u}_{h}\right)\right\} \cdot \mathbf{n} d S-\int_{\mathcal{E}_{b}} \phi_{h}^{+} \mathcal{F}_{v}\left(\mathbf{u}_{b}, \nabla \mathbf{u}_{b}\right) \cdot \mathbf{n} d S \\
& \left.-\int_{\mathcal{E}_{i}} \llbracket \mathbf{u}_{h} \rrbracket\left\{\mathbf{G}^{T}\left(\mathbf{u}_{h}\right) \nabla_{h} \phi_{h}\right\} \cdot \mathbf{n} d S-\int_{\mathcal{E}_{b}}\left(\mathbf{u}_{h}^{+}-\mathbf{u}_{b}\right)\left\{\mathbf{G}^{T}\left(\mathbf{u}_{b}\right) \nabla_{h} \phi_{h}^{+}\right)\right\} \cdot \mathbf{n} d S \\
& +\int_{\mathcal{E}_{i}} \llbracket \phi_{h} \rrbracket \boldsymbol{\delta}\left(\mathbf{u}_{h}\right) \cdot \mathbf{n} d S+\int_{\mathcal{E}_{b}} \phi_{h}^{+} \boldsymbol{\delta}_{b}\left(\mathbf{u}_{h}^{+}, \mathbf{u}_{b}\right) \cdot \mathbf{n} d S
\end{aligned}
$$


where $\mathbf{G}\left(\mathbf{u}_{h}\right)=\partial \mathcal{F}_{v}\left(\mathbf{u}_{h}, \nabla_{h} \mathbf{u}_{h}\right) / \partial\left(\nabla_{h} \mathbf{u}_{h}\right)$ is the so-called homogeneity tensor. The viscous fluxes may thus be written as $\mathcal{F}_{v}\left(\mathbf{u}_{h}, \nabla_{h} \mathbf{u}_{h}\right)=\mathbf{G}\left(\mathbf{u}_{h}\right) \nabla_{h} \mathbf{u}_{h}$. The penalty function is defined following the approach proposed in [8] as

$$
\boldsymbol{\delta}\left(\mathbf{u}_{h}\right)=\eta_{I P} \frac{p^{2}}{h} \mathbf{G}\left(\mathbf{u}_{h}\right) \llbracket \mathbf{u}_{h} \rrbracket
$$

with $\eta_{I P}$ the penalty parameter, which must be chosen sufficiently large.

The integrals in Eqns. (5) and (7) are computed by means of the GaussLegendre quadrature with $q=p+1+m$ points in each space direction, where $m$ depends on the test case considered.

As regards the SGS term, the effect of the subgrid scales can be approximated by a closure term that depends only on the resolved field, $\mathcal{L}_{s g s}\left(\mathbf{u}, \mathbf{u}_{h}, \phi_{h}\right) \approx$ $\mathcal{L}_{m}\left(\mathbf{u}_{h}, \phi_{h}\right)$. The compressible LES formalism of [11] is used here, which consists in introducing a SGS flux vector of the form,

$$
\mathcal{F}_{m}=\left(0, \overline{\overline{\boldsymbol{\tau}}}^{\text {sgs }},-\mathbf{q}^{\text {sgs }}\right)^{T}
$$

Using the eddy-viscosity assumption, the SGS stress tensor $\overline{\overline{\boldsymbol{\tau}}}^{\text {sgs }}$ is written as

$$
\tau_{i j}^{s g s}=\rho_{h} \nu_{t}\left(2 S_{i j}-\frac{2}{3} S_{k k} \delta_{i j}\right)
$$

where $\rho_{h}$ is the resolved density, $S_{i j}$ are the components of the resolved rate-ofstrain tensor $\overline{\overline{\mathbf{S}}}$, and $\nu_{t}$ the turbulent eddy viscosity. The SGS heat-flux vector is given by

$$
q_{i}^{s g s}=-\rho_{h} C_{p} \frac{\nu_{t}}{P r_{t}} \frac{\partial T_{h}}{\partial x_{i}}
$$

where $T_{h}$ denotes the resolved temperature and $P r_{t}$ is the turbulent Prandtl number, assumed to be constant and equal to 0.6.

In this work, the Smagorinsky model is used, for which $\nu_{t}$ is expressed as

$$
\nu_{t}=\left(C_{s} \Delta\right)^{2}|\overline{\overline{\mathbf{S}}}| ;|\overline{\overline{\mathbf{S}}}|=\sqrt{2 S_{i j} S_{i j}}
$$

In (12), $C_{s}$ is the model constant which usually takes a value between 0.1 and 0.2 , and $\Delta$ is the filter size associated with a given element, defined here as $\Delta=\frac{h}{p+1}$, with $h$ the characteristic size of the element.

Based on the formalism laid out above, the model term $\mathcal{L}_{m}\left(\mathbf{u}_{h}, \phi_{h}\right)$ is discretized using the same scheme employed to discretize the viscous fluxes. This amounts to replacing $\mathcal{F}_{v}$ by $\mathcal{F}_{m}$ in Eqn. (7). For more details on the DG-LES formalism implemented in Aghora the reader is referred to $[5,6]$.

In VMS we assume that the effect of the unresolved scales on the largest resolved scales is negligible and thus the effect of the SGS model is confined to a range of small resolved scales. This is obtained in the DG-VMS framework by splitting the polynomial solution space $\mathcal{V}_{h}^{p}$ into a low-order component $\mathcal{V}^{l} \equiv \mathcal{V}_{h}^{p_{L}} \subseteq \mathcal{V}_{h}^{p}$, associated with the large scales, and a high-order component $\mathcal{V}^{s} \equiv \mathcal{V}_{h}^{p} \backslash \mathcal{V}^{l}$ presenting the small scales, where $p_{L}$ is called the scale-partition parameter. In this work, the SGS term is computed from the full resolved field, as proposed in [5], and the effect of the SGS model is removed from all scales belonging to $\mathcal{V}^{l}$ by enforcing $\mathcal{L}_{m}\left(\mathbf{u}_{h}, \phi_{h}\right)=0, \forall \phi_{h} \in \mathcal{V}^{l}$. 


\section{DG-VMS of the flow past a circular cylinder}

The sections that follow report the results from the DG-VMS simulations of the cylinder flow at $R e=20000$ and 140000 . At these sub-critical Reynolds numbers the boundary layer separates laminarly from the cylinder surface and transition to turbulence takes place in the free-shear layer. It is worth noting that in the version of the VMS algorithm used in this work, the scale-partition parameter $p_{L}$ that sets the limit between large and small scales is constant across the domain. This implies that the SGS model will also be active in the cells covering the (laminar) boundary layer region. This is, however, not an issue in VMS, as, by definition, only the small scales are affected by the SGS dissipation, while the mean flow is let free of SGS dissipation. In the DG-VMS simulations presented in this paper a partition number $p_{L}=1$ has been used (see Sec. 3 and [5]).

In both simulations a curved O-type mesh is considered in a computational domain with radial and spanwise extension of $25 D$ and $\pi D$, respectively. The number of elements in the azimuthal and radial directions is 36 , and in the spanwise direction 16 elements are used. This amounts to a total number of elements equal to 20736 . In the case of the higher Reynolds number, a higher stretching factor is used in a region closer to the cylinder, which leads to a more pronounced clustering of the elements in the vicinity of the wall, with respect to the lower-Reynolds-number case. As will be shown below, this is consistent with the shorter length of the recirculation bubble expected and the resolution requirements of the boundary layer at this higher Reynolds number. The focus in this work is therefore on capturing the flow dynamics in the near-wake behind the cylinder, by putting most of the computational effort in this region. The region beyond the recirculation area can thus be considered as under-resolved.

Periodicity of the flow is assumed in the spanwise direction, and an isothermal no-slip boundary condition is imposed on the cylinder wall. A laminar freestream flow at $M=0.2$ is imposed via a far-field condition on the outer boundary of the computational domain. Finally, the time integration is performed using an explicit third-order accurate Runge-Kutta method.

\subsection{Simulation results at $R e=20000$}

For this lower-Reynolds-number case, the polynomial degree is set to $p=4$, which leads to fifth-order accuracy in space and a number of DOFs of 2.59 million (Mdofs). The effective resolution at the wall is $\Delta r /(p+1)=0.01 D$.

Over-integration is used to reduce aliasing errors and increase the accuracy of the solution. A horizontal uniform flow at $\mathrm{M}=0.2$ is used as initial condition. After a transient period, a statistically steady (periodic) state is reached, from which the flow statistics are gathered for approximately 95 vortex-shedding cycles. Table 1 compares our DG-VMS results with those obtained by Lysenko et al. [13] using a second-order low-dissipative FV compressible solver at the same Mach number $M=0.2$ and for the same dimension of the computational domain as in the present simulation. The main integral flow quantities are shown in this table. The range of values shown for the reference simulation correspond 
Table 1. Cylinder flow at $R e=20$ 000: integral flow quantities.

\begin{tabular}{llllllll}
\hline Simulation & $\frac{\Delta r_{\min }}{D}$ & Mdofs & $N_{v s}$ & $C_{d}$ & $C l_{r m s}$ & $S_{t}$ & $\frac{L_{r}}{D}$ \\
\hline$F V-\mathcal{O}(2)[13]$ & $5.610^{-4}$ & 12.4 & 75 & $1.33-1.36$ & $0.61-0.70$ & $0.18-0.19$ & $0.57-0.69$ \\
$D G-\mathcal{O}(5)$ & $10^{-2}$ & 2.59 & 95 & 1.39 & 0.64 & 0.19 & 0.67 \\
\hline
\end{tabular}
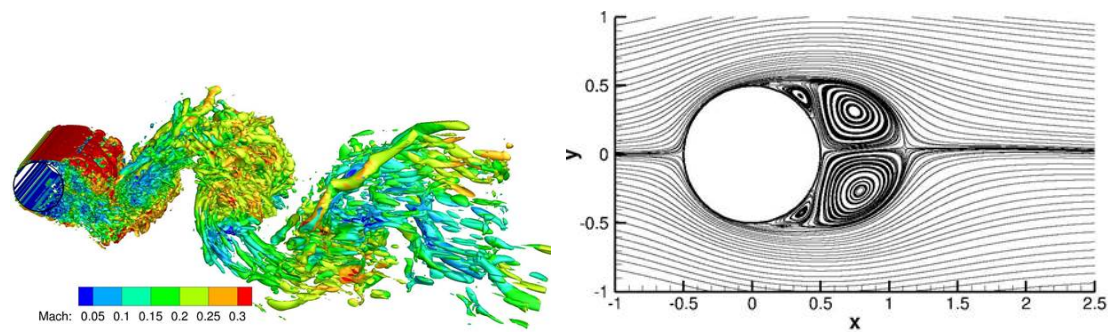

Fig. 1. Flow past a circular cylinder at $R e=20000$. Left: iso-surfaces of the Q-criterion coloured by the Mach number. Right: streamlines of mean flow.

to the use of different numerical schemes and SGS modelling approaches. More details about the FV simulations by Lysenko et al. can be found in their original paper [13].

We can see from this table that the results yielded by the DG-VMS fifth-order simulation are in fairly good agreement with the reference LES results. Only the value of the drag coefficient $C_{d}$ is slightly above the upper-most value of the reference range, by about $2.2 \%$. This is despite the fact that the DG simulation involves nearly five times fewer DOFs than the FV simulation. It is worth noting the much smaller effective size of the grid cells at the wall, which in the DG simulation is about 18 times smaller with respect to the reference value. The good prediction achieved with our DG approach is partly a consequence of the excellent approximation properties of polynomial expansions in representing thin boundary layers, as pointed out by Gottlieb and Orszag [7]. This coarseness of the mesh in the vicinity of the wall does not seem to impact the accuracy with which the integral quantities, and in particular the size of the recirculation bubble $L_{r}$, are captured. In fact, the value of $L_{r}$ is well within the range of values reported in [13]. Figure 1 shows a snapshot of the iso-surfaces of Q-criterion coloured by the Mach number (left) as well as the streamlines of the time-averaged flow field (right). We can see that the mean flow is characterised by the presence of a main recirculation bubble, and the appearance of two symmetrical secondary bubbles near the separation point in accordance with the literature. The value of the primal separation angle is estimated from the profile of the wall-shear stress, which leads to $\theta_{\text {sep }}=88^{\circ}$. This estimated value is in good agreement with the results of Lysenko et al. who predicted from their LES a value in the range $\theta_{\text {sep }}=86^{\circ}-88^{\circ}$. As pointed out by the author, these values are about $13 \%$ above the value predicted in the experiments of Son and Hanratty [20] and 

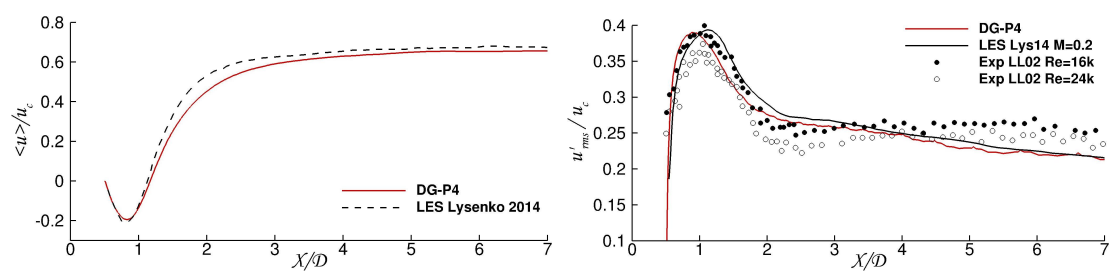

Fig. 2. Cylinder flow at $R e=20000$ : streamwise velocity statistics on wake centreline. Left: mean streamwise velocity; solid line: $D G-p 4$, dashed line: incompressible FV simulation (TKE-I in [13]). Right: rms of streamwise velocity; red solid line: $D G-p 4$, black solid line: compressible FV simulation (SMAG- $\gamma$ in [13]), symbols: experimental data of Lim and Lee [12] at $R e=16000$ (dots) and $R e=24000$ (circles).

Norberg [16]. The cause for these discrepancies might be an insufficient resolution of the boundary layer, or else possible differences between the simulation and the experimental conditions.

To further assess the quality of our results, the statistics in the near-wake region are also analysed. Figure 2 therefore shows the mean streamwise velocity along the wake centreline (left), as well as the profile of root-mean-square (rms) of the axial velocity fluctuations (right). The present results are compared with the available data from [13]. For the first, the only data available is that from an incompressible FV simulation using the dynamic $k$-equation SGS model (see [13] for more details). Despite the different equation models used, the two mean streamwise velocity profiles are in relatively good agreement. As regards the profiles of rms of the axial velocity, our results are compared with the compressible CFD results from [13] on the fine mesh and the experimental data of Son and Hanratty [20] for Reynolds numbers $R e=16000$ and 24000 . We can see that, overall, our results are in rather good agreement with the reference numerical data, and well within the range of the values predicted by the experiment. However, the departure from the experiment beyond approximately $x / D=4$ is obvious from this plot, and equally visible in the reference CFD data. This is partly a consequence of the lack of resolution in this region. It is interesting to note that this under-resolution does not seem to have a strong effect on the quality of the prediction in the wall region and near-wake dynamics.

\subsection{Static p-adapted simulation results at $R e=140000$}

The use of p-adaptation, involving refinement as well as coarsening, allows for an important reduction of the number of DOFs with respect to uniform refinement, as well as an increased level of accuracy.

\section{Description of the p-adapted algorithm}

The p-adaptive algorithm employed here is that developed in [15]. The algorithm is initialised by specifying a uniform polynomial degree distribution. A first 

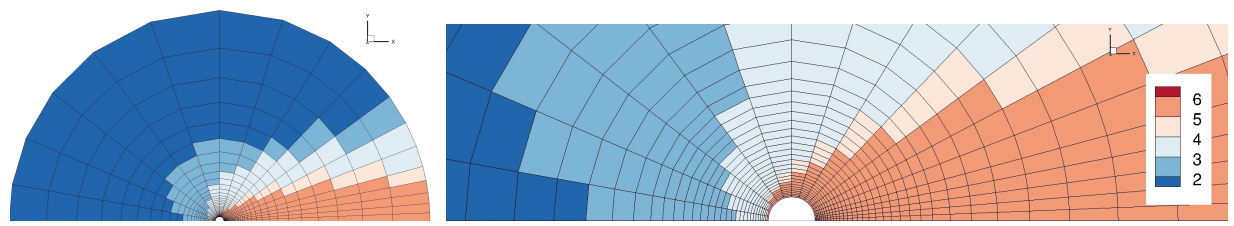

Fig. 3. p-adapted DG-VMS of cylinder flow at $R e=140000$ : distribution of p.

simulation is then carried out based on the prescribed polynomial degree that will serve as initial solution for the next iteration of the p-adaptive algorithm. At each iteration of the algorithm, a new distribution of polynomial degrees is defined based on local error estimates and a new numerical solution is computed. The error estimator used in this work is the small-scale energy density (SSED) estimator proposed in [15], which can also be interpreted as a measure of the kinetic energy associated with the highest-order modes representing the solution within the element.

For the simulations presented here, an initial uniform distribution of polynomial degree $p=4$ is used. The DG simulation is then run until the flow is fully developed and reaches a statistically periodic state. From this point, the element-wise values of the SSED estimator are computed over approximately five vortex-shedding cycles.

Based on the p-adaptation strategy just described, a new distribution of polynomial degrees is obtained. A new iteration of the p-adaptation algorithm is then applied. In this work, two iterations of the adaptive algorithm have been performed leading to the p-pattern shown in Fig. 3, with $p$ varying from 2 to 6 . In the spanwise direction $p$ is kept constant. This is consistent with the statistically $2 \mathrm{D}$ character of the flow. The polynomial degree in the first element layer around the cylinder is $p=6$. This yields an effective size of the first element at the wall of $\Delta r /(p+1)=4 \cdot 10^{-3}$. This value is about 2.5 smaller than that corresponding to $R e=20000$, in accordance with the fact that the boundary layer thickness is inversely proportional to $\sqrt{R e}$ as predicted by the theory.

$D G-V M S$ results at $R e=140000$

The p-adapted DG solution at this higher Re is compared with the numerical data reported by Breuer [2], as well as the experimental data from Cantwell and Coles [3]. In [2], the author performed a number of simulations using different levels of resolution and spanwise extensions of the computational domain. Here we consider for comparison the simulations $C 3$ and $D 3$ from [2] corresponding to a spanwise length of the cylindrical domain of $\pi D$ and a radial extension of $15 D$. The number of DOFs was 1.74 and 6.76 DOFs, respectively, for simulations $C 3$ and D3. They are both based on the Smagorinsky model for SGS modelling.

Table 2 compiles the values of the integral quantities obtained from the present simulation and for the reference data used for comparison. As regards the values of drag, $C_{d}$, and base pressure, $-C_{p, b}$, coefficients, our results are in good 
Table 2. Circular cylinder flow at $R e=140000$ : comparison of integral flow quantities with the simulation results of Breuer [2] and Cantwell and Coles [3].

\begin{tabular}{lllllllll}
\hline Data & $L_{z} / D$ & Mdofs & $\frac{\Delta r_{\min }}{D}$ & $N_{v s}$ & $C_{d}$ & $-C_{p, b}$ & $S_{t}$ & $\frac{L_{r}}{D}$ \\
\hline Experiment [3] & - & - & - & - & 1.24 & 1.21 & 0.18 & 0.50 \\
Breuer-C3[2] & $\pi$ & 1.74 & $410^{-4}$ & $13-34$ & 1.28 & 1.51 & 0.22 & 0.46 \\
Breuer-D3[2] & $\pi$ & 6.76 & $410^{-4}$ & $13-34$ & 1.37 & 1.60 & 0.21 & 0.42 \\
DG-O $(3-7)$ & $\pi$ & 4.98 & $410^{-3}$ & 71 & 1.43 & 1.59 & 0.19 & 0.50 \\
\hline
\end{tabular}
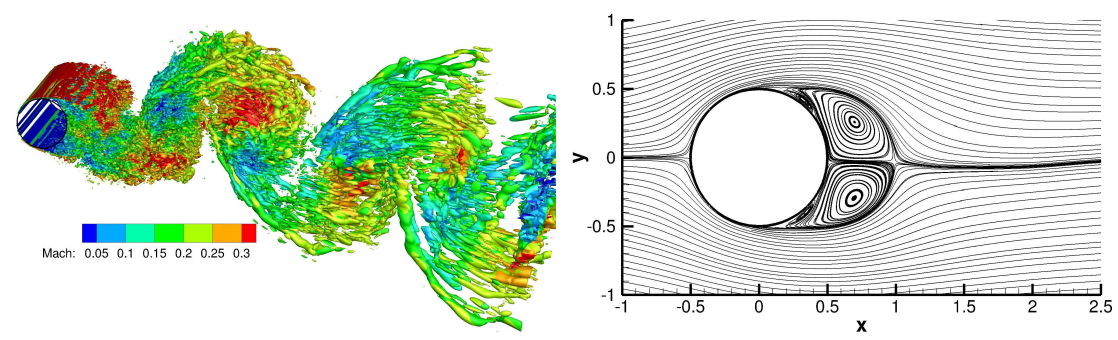

Fig. 4. Cylinder at $R e=140000$. Left: iso-surfaces of the Q-criterion coloured by the Mach number. Right: streamlines of mean flow.

agreement with the CFD data from Breuer, though substantially larger than the experimental value. The Strouhal number, $S_{t}$, is in between the experimental and the numerical reference values. The $S_{t}$ measured by Cantwell and Coles [3] appears, however, very low with respect to what has been found in other experimental works. In particular, Son and Hanratty [20] report a value slightly above 0.19 which is consistent with the value found in the present work. Finally, the recirculation length, $L_{r}$, predicted by the DG simulation is in perfect agreement with the experimental value and slightly higher than the numerical reference. We have however observed, that the value of this quantity is very sensitive to the averaging period, in agreement with the observations made in [17]. This might explain the differences found between our value and that reported by Breuer, who considered an averaging time considerably lower. As pointed out in [17], and from our own observations, this quantity is actually a very reliable indicator of the level of accuracy in the simulation. It is indeed very sensitive to the mesh resolution in the near-wake region, as well as to the amount of numerical and SGS dissipation in the simulation.

Figure 4 shows a snapshot of the iso-surfaces of Q-criterion coloured by the Mach number (left) and the streamlines of the time-averaged flow field (right). We can observe the laminar separation that takes place on the cylinder surface and the significantly larger-scale vortex shedding originating from this separation, as compared to the case at $R e=20000$. The separation angle is estimated to be about $94^{\circ}$ in agreement with the value reported by Breuer for simulation $C 3$ on the coarser mesh, and slightly higher than the value $92^{\circ}$ obtained by the 

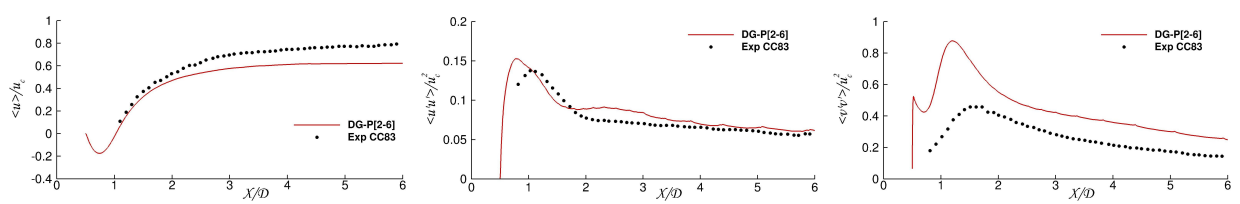

Fig. 5. Flow past a circular cylinder at $R e=140000$. Wake centreline statistics. • : Experiments [3], red solid line: p-adapted DG-VMS.
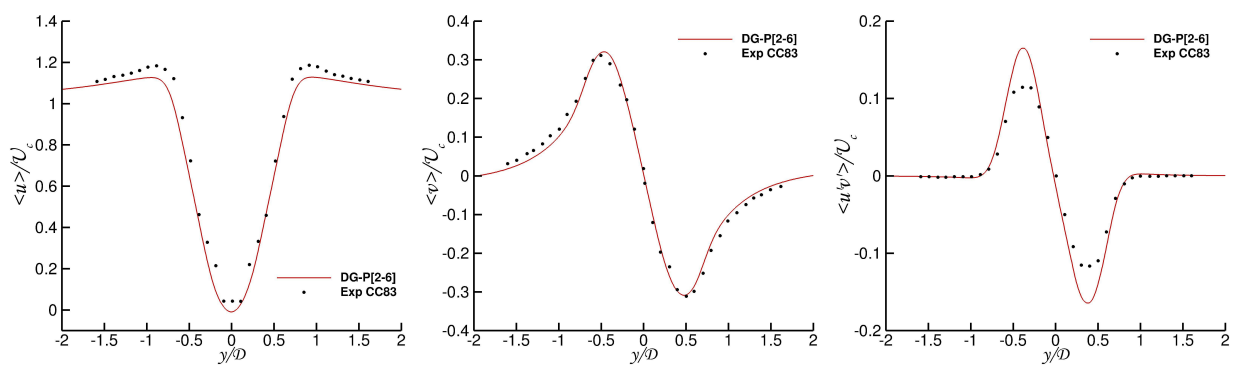

Fig. 6. Flow past a circular cylinder at $R e=140000$. Wake statistics at $\mathrm{x}=1$. • : Experiments [3], red solid line: p-adapted DG-VMS.

author on the finer grid (simulation D3). On the right, we can see the plots of the streamlines of the mean flow. The most salient feature is the length of the recirculation bubble that shrinks as the Re number is increased. It is also worth noting the two small secondary bubbles showing up in our simulation, not observed in the experiments, nor in the simulation by Breuer. These two small secondary bubbles have also been found, however, in the simulation performed by Karabelas [10]. No clear explanation for this discrepancy with the experiments can be given today, and further research will be necessary to clarify this point. As regards the $r m s$ of the drag and lift coefficients we obtain the following values, $C_{d}^{\prime}=0.094$ and $C_{l}^{\prime}=0.64$. For this particular Reynolds no values for these quantities have been reported in the literature.

In Fig. 5, the wake centreline statistics are also compared with those obtained from the experiment. As regards the mean velocity profile, we can see that the length of the recirculation region matches very well the experimental value (see also Table 2). It is apparent from these results, however, that as we move further downstream from the recirculation region, the streamwise velocity levels are significantly under-predicted. This is partly due to the very coarse mesh used in the wake beyond the recirculation region. This is in contrast with the profiles of rms of the streamwise velocity $\left\langle u^{\prime} u^{\prime}\right\rangle$, better predicted over a longer distance beyond the recirculation zone. This might be a consequence of the higher levels, with respect to the reference, found in the region closer to the cylinder base. As regards the cross-stream Reynolds stresses $\left\langle v^{\prime} v^{\prime}\right\rangle$, their magnitude is significantly higher than the experimental value, although in fairly agreement with the values predicted by Breuer on his fine-grid simulations (see [2], not shown here). We 
have also inspected the wake statistics at a location $x=1$ and compared to the experimental data of Cantwell and Coles [3]. The outcome from this comparison can be seen in Fig. 6. We can observe the overall good agreement found with the experiment for these quantities. The most significant discrepancies appear in the profile of the shear stresses $\left\langle u^{\prime} v^{\prime}\right\rangle$, although their magnitude is in line with the differences found by Breuer on his fine-grid simulations. These discrepancies might be linked to the different conditions between the experiment and the simulations (e.g. zero turbulence level at the inlet in the simulation). In fact, regarding the study carried out in [2], the author mentioned that grid refinement did not appear to provide convergence towards the experimental values.

\section{Conclusions}

The scale-resolving capabilities of the DG solver Aghora [4,18] have been illustrated by performing high-order LES of the flow past a circular cylinder in the sub-critical regime. The SGS modelling approach adopted here is based on the DG-VMS formalism described in [5]. The static p-adaptive algorithm recently proposed in [15] has been successfully applied to cylinder flow at $R e=140000$. The maximum order of accuracy used in these simulations amounts to fifth and seventh-order for $R e=20000$ and 140000 , respectively.

The outcome of our simulations has been compared with the high-resolution LES results reported by Lysenko et al. [13] for $R e=20000$, and by Breuer [2] for $R e=140000$, using low-dissipation second-order FVM, as well as with the available experimental data. An overall good agreement with the reference CFD data is found in terms of integral flow quantities and near-wake statistics. This is despite the fact that the DG-VMS simulations involve much fewer DOFs than the FV LES. For the higher Re, some discrepancies with the experimental data have been found. These differences are, however, in line with those found in the work of Breuer [2] and, based on published research, might be explained by the different flow conditions between the simulations and the experiment.

It is noteworthy that the effective height of the first grid cell off the wall in the DG simulations is one order of magnitude larger than in the reference LES. This highlights the excellent approximation properties of DG approximations in representing boundary layers on coarse grids.

\section{Acknowledgements}

This research has been partly funded by the European Union's Horizon 2020 TILDA project (grant agreement number 635962). This work was performed using HPC resources from GENCI (Grants A0022A10129 and A0032A10309).

\section{References}

1. Bassi F., Botti L., Colombo A., Di Pietro D.A., Tesini P. (2012) On the flexibility of agglomeration based physical space DG discretizations, J Comput Phys 231(1):4565 . 
2. Breuer, M. (2000) A challenging test case for large-eddy-simulation: high Reynolds number circular cylinder flow, Int J Heat Fluid Flow 21(5):648-654.

3. Cantwell, B. and Coles, D. (1983) An experimental study of entrainment and transport in the turbulent near wake of a circular cylinder, J Fluid Mech 136:321-374.

4. Chapelier, J.B., de la Llave Plata, M., Renac, F., Lamballais, E. (2014) Evaluation of a high-order discontinuous Galerkin method for the DNS of turbulent flows, Comput Fluids 95, pp. 210-226.

5. Chapelier J.B., de la Llave Plata M., Lamballais E. (2016) Development of a multiscale LES model in the context of a modal DG method, Comput Meth Appl Mech Eng 307:275-299.

6. de la Llave Plata, M., Couaillier, V., Le Pape, M.C. (2018) On the use of a highorder discontinuous Galerkin method for DNS and LES of wall-bounded turbulence, Comput Fluids 176:320-337.

7. Gottlieb, D. and Orszag, S.A., Numerical analysis of spectral methods : theory and applications, SIAM, 1977.

8. Hartmann, R. and Houston, P. (2008) An optimal order interior penalty DG discretization of the compressible Navier-Stokes equations, J Comput Phys 227(22):9670-9685.

9. Fröhlich J., Rodi W., Bertoglio J.P., Bieder U., Touil H. (2001) LES of flow around circular cylinders on structured and unstructured grids, II. In: Hirschel E.H. (eds) Numerical Flow Simulation II. Notes Numer Fluid Mech 75, Springer.

10. Karabelas, S.J., LES of high-Reynolds number flow past a rotating cylinder (2010) Int J Heat Fluid Flow 31(4):518-527.

11. Lesieur, M., Métais, O. and Comte, P., Large-eddy simulation of turbulence, Cambridge University Press, Cambridge 2005.

12. Lim, H.C. and Lee, S.J. (2002) Flow control of circular cylinders with longitudinal grooved surfaces, AIAA J. 40(10):2027-2036.

13. Lysenko, D.A., Ertesvåg, I.S. and Rian, K.E. (2014) LES of the flow over a circular cylinder at Reynolds number $2 \times 10^{4}$, Flow Turbul Combust 92(3):673-698.

14. Moussaed, C., Wornom, S., Salvetti, M.-S., Koobus, B. and Dervieux, A. (2014) Impact of dynamic subgrid-scale modeling in variational multiscale large-eddy simulation of bluff-body flows, Acta Mech 225(12):3309-3323.

15. Naddei, F., de la Llave Plata, M. and Couaillier, V. (2019) A comparison of refinement indicators for p-adaptive simulations of steady and unsteady flows using DG methods, J Comp Phys 376:508-533.

16. Norberg, C. (2003) Fluctuating lift on a circular cylinder: review and new measurements, J Fluids Struct 17:57-96.

17. Parnaudeau P., Carlier J., Heitz D., Lamballais E. (2008) Experimental and numerical studies of the flow over a circular cylinder at Reynolds number 3900. Phys Fluids 20(8):085101

18. Renac F., de la Llave Plata M., Martin E., Chapelier J.-B., Couaillier C.: Aghora: A High-Order DG Solver for Turbulent Flow Simulations. In: Kroll et al. (eds.) Notes Numer. Fluid Mech. Multidisc. Design, vol. 128:315-335, Springer, 2015.

19. Salvatici, E. and Salvetti, M.V. (2003) Large-eddy simulations of the flow around a circular cylinder: effects of grid resolution and subgrid scale modeling, Wind and Structures, 6(6):419-436.

20. Son, J. and Hanratty, T. (1969) Velocity gradients at the wall for flow around a cylinder at Reynolds numbers from $5 \times 10^{3}$ to $10^{5}$, J Fluid Mech 35:353-368. 